\title{
La enfermedad de Graves, signos y síntomas
}

\author{
P. YOUNG, B. C. FINN, J. E. BRUETMAN \\ Servicio de Clínica Médica. Hospital Británico. Buenos Aires, Argentina
}

GRAVES' DISEASE, SIGN AND SYMPTOMS

\begin{abstract}
RESUMEN
La enfermedad de Graves es la causa más común de hipertiroidismo, es de patogenia autoinmune. Se distingue clínicamente de otras formas de hipertiroidismo por la presencia de bocio difuso, oftalmopatía y ocasionalmente mixedema pretibial. En este artículo describimos la vida y obra de Robert Graves, realizando posteriormente una revisión de los signos y síntomas de la enfermedad. En el mundo de la medicina actual, en donde la tecnología juega un rol preponderante, queremos recordar la importancia de la anamnesis y el examen físico como herramienta indispensable del clínico.
\end{abstract}

PALABRAS CLAVE: Hipertiroidismo. Enfermedad de Graves. Historia de la medicina.

\begin{abstract}
Graves'disease is by far the most common cause of hyperthyroidism. Is an immunologic disorder and it is distinguished clinically from other forms of hyperthyroidism by the presence of diffuse thyroid enlargement, ophthalmopathy, and occasionally pretibial myxedema. In this paper we summarize the prolific life of Robert Graves and we also describe the signs and symptoms of hyperthyroidism. In today's medicine, were technology plays a very important role, we would like to remark the value of anamnesis and physical exam as some of the most useful tools.
\end{abstract}

KEY WORDS: Hyperthyroidism-Graves' disease. History of medicine.

Young P, Finn BC, Bruetman JE. La enfermedad de graves, signos y síntomas. An Med Interna (Madrid) 2007; 24: $505-508$.

\section{INTRODUCCIÓN}

Robert James Graves nació en Dublín en 1796 (1). Se graduó en medicina en el Trinity College de Dublín a la edad de 22 años. Obtuvo el primer lugar en su clase por lo que recibió la medalla de oro, luego de su graduación en 1818, comenzó un recorrido por diversos hospitales europeos, visitando Londres, Gottingen, Berlin, Copenhague, y Edimburgo. En Alemania recibió la influencia de los métodos de enseñanza clínica. Viajó también por Italia en donde desarrollo una profunda amistad con el famoso pintor Joseph Mallord William Turner (1), compartiendo sus cualidades artisticas.

En 1821 Graves vuelve a Dublín e ingresa como médico en el Meath Hospital, convirtiéndose en los siguientes 15 años, en un extraordinario médico y profesor. Un día típico en la vida de Graves, comenzaba en las salas del hospital a las 7 am a la luz de las velas, luego realizaba visitas a domicilio. Diariamente a las 4 pm enseñaba clínica a aproximadamente 100 estudiantes en un anfiteatro. Probablemente su mayor contribución fue el desarrollo de una forma sistemática de enseñar junto a la cama del paciente. Puso énfasis en la obser- vación detallada y en el conocimiento de enfermedades crónicas comunes, con especial interés en las enfermedades infecciosas y la fiebre, así como también un enfoque humanitario de los pacientes.

Formo parte de un grupo de médicos que combatieron la epidemia de fiebre tifoidea en el oeste de Irlanda (1).

En 1824 Graves, fue cofundador de Park Street School of Medicine. Por este tiempo realizó observaciones originales, se cree que fue el primero que describió el edema angioneurótico y realizó la primera publicación de las características clínicas de la enfermedad de Raynaud. Publicó en múltiples revistas y escribió en 1843, un libro titulado A system of clinical medicine, basado en los ateneos del Sir Patrick Duns Hospital $(1,2)$.

$\mathrm{Su}$ vida personal no fue tan satisfactoria como la profesional. Se caso por primera vez en 1821, su esposa Matilda Jane y su hijo fallecieron en 1825. Un año más tarde se caso nuevamente con Sarah Jane, que también fallece junto al hijo de ambos al siguiente año. Se caso por tercera vez en 1830 con Ann Grogan, con la cual tuvo seis hijos $(1,2)$.

También desarrollo una intensa labor en la escuela de medicina del Trinity College. Fue elegido presidente del Royal

Trabajo aceptado: 4 de ocubre de 2007 
College of Physicians de Irlanda en 1843, el mismo año que dejo el Meath Hospital $(1,2)$.

Se sabe poco de sus últimos años, fallece el 20 de marzo de 1853, a la edad de 57 años de un tumor abdominal.

Graves fue uno de los mayores exponentes de la edad de oro de la medicina Irlandesa, lo demuestran sus contemporáneos como son Stokes, Adams, Cheyne, Todd, Colles, Corrigan, y Sir William Wilde, famoso otorrinolaringólogo de las Islas Británicas y padre de Oscar Wilde.

Robert J. Graves es recordado hoy, por las múltiples contribuciones en el desarrollo de la enseñanza clínica, sus observaciones originales y su prolífica labor como escritor de artículos médicos, respetado en su tiempo por el reconocido Trousseau en Paris y posteriormente por Osler $(1,2)$.

Uno de sus artículos, Newly observed affection of the thyroid gland in females, fue publicado en el London Medical and Surgical Journal en 1835 (2). Fue en este artículo donde realiza un pormenorizado detalle de las características clínicas de lo que ahora se conoce como enfermedad de Graves (EG), aunque esta ya había sido descripta previamente por Caleb Hillier Parry en 1825 (1-3).

Fue Graves fue el primero en identificar la triada de bocio, palpitaciones y exoftalmos (3).

En Alemania se adjudica la descripción de las características clínicas de la enfermedad a Carl Adolph von Basedow que vivió en el mismo tiempo que Robert Graves. Tan es así que se le adjudica haber descrito lo que se ha dado a llamar triada de Merseburg conformada por exoftalmos, bocio y taquicardia similar a la descripta por Graves. La dermopatía tiroidea en Alemania se llama enfermedad de Basedow (4).

Otras denominaciones de la EG son: enfermedad de March, enfermedad de Parsons, enfermedad de Flajani, enfermedad de Begbie, enfermedad de Graves-Basedow-Parry.

Hoy día se sabe que es un desorden autoinmune, caracterizado por la producción de inmunoglobulinas de tipo $\mathrm{IgG}$, que estimulan el receptor de la hormona que estimula la tiroides (TSH) $(1,3)$. Estas inmunoglobulinas son también dirigidas contra los tejidos blandos de la órbita lo que produce edema e inflamación, con proptósis, edema periórbitario y oftalmoplejía. Esta asociación entre hipertiroidismo y enfermedad ocular, resulta de la presencia de proteínas receptoras TSH-like dentro del músculo y la grasa orbitaria. Otros signos extratiroideos del proceso autoinmune son el mixedema pretibial y la acropaquia $(1,3)$.

Entre los pacientes con hipertiroidismo el 60 a $80 \%$ corresponde a EG, dependiendo de factores regionales como la ingesta de iodo (3). La incidencia anual en mujeres es de 0,5 por 1000 , con una edad de comienzo entre los 40 a 60 años. La relación mujer/hombre es de 8/1. Es el desorden autoinmune más prevalente de los estados unidos (3).

El espectro clínico es complejo ya que existen pacientes con anticuerpos que estimulan el receptor de TSH o lo pueden bloquear (Anticuerpos bloqueantes). Como resultado de ello existe un rango de expresión clínica desde hipertiroidismo al eutiroidismo o hipotiroidismo e hipertiroidismo recurrente que depende de la actividad de anticuerpos que estimulen o bloqueen el receptor de TSH $(1,3)$.

A menudo las manifestaciones clínicas se diferencian en signos o síntomas de hipertiroidismo no complicado, EG propiamente dicha, condiciones asociadas a la EG y la tirotoxicosis $(1-3,5,6)$.
Los síntomas más comunes del hipertiroidismo no complicado son el nerviosismo, fatiga, palpitaciones, intolerancia al calor y pérdida de peso que se encuentran en más de la mitad de los pacientes (3). Cabe resaltar que aumentan de peso un $10 \%$ de los pacientes (3). Síntomas menos comunes son disnea, prurito, dispepsia, vómitos y diarrea $(3,5,6)$. Raros son la sialorrea, sed, poliuria y oligo o amenorrea con pérdida de la libido $(3,6)$. En algunos hipertiroideos se presentan crisis de vómitos, se ha dado a llamar forma emética del hipertiroidismo, a veces difícil de diagnosticar si los síntomas tiroideos son poco acentuados (6). También la diarrea puede ser el único signo de un hipertiroidismo, ésto se ha denominado forma enterótropa de la EG. Se llama síndrome neurocirculatorio de Labbé a la neurosis de ansiedad que se observa en esta entidad.

La taquicardia sinusal es, tal vez, el signo clínico más precoz y constante del hipertiroidismo, como precisó Charcot.

Aparte de la taquicardia otros signos más comunes son la fibrilación auricular, temblor fino, hiperreflexia, incremento de la sudoración y calor de la piel, eritema palpar (palma de Lane) (3). Signos menos comunes son la onicolísis, pérdida del cabello, debilidad muscular y caquexia $(3,6)$. En las formas severas de hipertiroidismo existe atrofia muscular difusa, a veces muy marcada, con intensa astenia, se la ha dado a llamar "forma muscular de Zondek" (6). Raros la insuficiencia cardiaca congestiva de alto gasto, corea, parálisis periódica y la psicosis $(3,5,6)$.

La fibrilación auricular es rara por debajo de los 50 años, pero ocurre en el $20 \%$ de los mayores de esta edad (3).

En algunos casos existe canicie precoz, en donde los cabellos son finos, escasos y encanecidos prematuramente, especialmente en la frente, por lo que se ha acuñado la frase "ceniza en la frente sobre el fuego de la mirada" (7).

Aparte de su signo, Sainton ha descrito que existe canicie en la región temporal de uno o ambos lados, se ha denominado "mechón hipertiroideo de Sainton" (7).

Las uñas en cuchara o coiloniquia (contorno cóncavo con onicolísis distal) se las encuentra en el 5\% de los pacientes hipertiroideos y se las denomina "uñas de Plummer" (3,5-7).

La asociación de hipertiroidismo con corea ha sido descripta por sutherland en Brain en 1903.

Dentro de las manifestaciones de la EG propiamente dicha se encuentran el bocio difuso, la oftalmopatía, la dermopatía localizada, la hiperplasia linfoide y la acropaquia tiroidea (3).

Aproximadamente el 90\% de los pacientes menores de 50 años tienen bocio difuso firme de tamaño variable, presentándolo el $70 \%$ de los mayores a esta edad (3).

Al hipertiroidismo que se presenta en manera de paroximos se le ha llamado síndrome de Lévi. La oftalmopatía clínica ocurre en alrededor del $50 \%$ de los pacientes, el $75 \%$ de las veces aparece dentro del año, antes o después del hipertiroidismo. Los hombres mayores tienen un alto riesgo de oftalmopatía severa. El 90\% de los pacientes tienen hipertiroidismo, el resto hipo o eutiroidismo. Clínicamente la oftalmopatía se manifiesta con retracción del párpado superior con mirada inquieta y brillante, sensación molesta en el ojo, dolor o presión retrobulbar, demora en el cierre palpebral, edema periorbitario, quemosis e inyección escleral. La mirada adquiere un aspecto de terror (ojo trágico), a veces tan acentuada que el ojo llega a subluxarse (subluxación de Dieulafoy). El exoftalmos ocurre en el $25 \%$ de los casos y la diplopía en el $10 \%$. También se puede ver disfunción de los músculos extraoculares, queratitis por exposición y neuropatía óptica. La enoftal- 
mía es rara $(1,3,6)$. A veces se acompaña de tumefacción orbitaria y paresia o parálisis de los músculos extrínsecos también llamado exoftalmos oftalmopléjico de Brain, y de dolor sordo en la órbita, signo grave, pues indica tumefacción orbitaria, con distensión subsiguiente de los tejidos alrededor del globo ocular.

La dermopatía ocurre en el 1 a $2 \%$ de los pacientes con EG, casi siempre en presencia de oftalmopatía severa, y se localiza principalmente a nivel pretibial (3).

Las manifestaciones extratiroideas de la EG no mejoran con el tratamiento dirigido al hipertiroidismo. En un subgrupo importante de pacientes con oftalmopatía tiroidea tienen remisión espontánea con el tiempo $(1,3)$.

No se deben olvidar las manifestaciones clínicas de las condiciones asociadas a la EG, como son la diabetes mellitus tipo 1, enfermedad de Addison, vitíligo, enfermedad de Addison Biermer, alopecia areata, miastenia gravis, y enfermedad celiaca entre otras (3).

En esta entidad se ha descrito como síndrome de ThomasDiamond al cuadro conformado por exoftalmia, mixedema pretibial y osteoartropatía hipertrófica (7).

La crisis tirotóxica o tirotoxicosis severa puede definirse como la repentina exacerbación de las manifestaciones del hipertiroidismo no complicado o tirotoxicosis que ponen en peligro la vida. Si bien no hay criterios uniformes para definir el momento en que una tirotoxicosis severa se convierte en una tormenta tiroidea, signos como hipertermia, taquicardia intensa, disfunción gastrointestinal y un cuadro psíquico de marcada excitabilidad, avalan la presencia de esta entidad (5). Existen formas atípicas de presentación de la tormenta tiroidea como son el coma, estado epiléptico, infarto cerebral no embólico, hipertermia, dolor abdominal e insuficiencia renal con rabdomiolisis (5).

Se han descrito tres formas clínicas de presentación de tirotoxicosis severa, la forma clásica, cardiovascular y la apática de Lahey (5).

En la forma clásica al hipertiroidismo no complicado se le agrega fiebre. La intolerancia al calor y la diaforesis comunes en el hipertiroidismo no complicado, se manifiestan en la crisis tirotóxica con severa hiperpirexia, superando aún los $42^{\circ} \mathrm{C}$. La marcada taquicardia ocurre en todos los casos, en forma desproporcionada a la hipertermia. La taquicardia sinusal leve a moderada del hipertiroidismo no complicado es reemplazada por importante taquicardia, a menudo mayor de 140 latidos por minuto y gran tendencia a la arritmia, como así también a grados variables de disfunción ventricular, insuficiencia cardiaca y edema pulmonar. Inicialmente la presión arterial se mantiene en valores normales o hipertensión sisto-diastólica transitoria que progresa hacia hipotensión y colapso, falleciendo de no mediar tratamiento precoz. Las manifestaciones del sistema nervioso central son ansiedad, temblor y fatiga comunes en los cuadros de hipertiroidismo no complicado a lo que se agrega agitación severa, delirio, confusión o franca psicosis, progresando en algunos casos a apatía, estupor y coma. Dentro de las manifestaciones gastrointestinales se encuentran el aumento del ritmo evacuatorio y ascenso de las transaminasas. El paciente presenta náuseas, vómitos, franca diarrea con desnutrición, deshidratación y dolor abdominal, que pueden sugerir un abdomen agudo. La hepatomegalia es frecuente y puede observarse marcada disfunción hepatocelular evidenciable por ictericia (5).

La forma apática fue descripta inicialmente por Lahey en
1931, más frecuente en pacientes añosos, y se caracteriza por ausencia de bocio prominente o exoftalmos y postración extrema con apatía, somnolencia, hipotonía muscular, cuadriparesia y ocasionalmente paresia de los músculos faciales y orofaringoláringeos (8).

En la forma cardiovascular predominan los síntomas secundarios a la insuficiencia cardiaca con fibrilación o aleteo auricular y arritmias ventriculares. El ECG muestra trastornos de repolarización secundarios a isquemia o infarto. En la actualidad, el uso de beta bloqueantes en patologías cardiovasculares puede enmascarar signos de toxicidad, por lo que se debe sospechar el diagnóstico en pacientes con estado confusional o con angina que no cede con tratamiento bloqueante (5).

Se ha descrito también como forma de presentación de tiroxicosis el síndrome de Kocher que consta de leucopenia por neutropenia, con linfocitosis relativa y absoluta debida a eosinofilia.

Así como el exoftalmos, es manifestación cardinal de la enfermedad, se pueden encontrar ciertos hallazgos en el examen físico en algunos de estos pacientes. Entre ellos recordamos los siguientes, por orden alfabético:

Signo de Abadi: Espasmo del elevador del párpado superior.

Signo de Boston: Cierre abrupto del párpado superior al rotar el ojo hacia abajo.

Signo de Bryson: Disminución en el poder de ampliación del tórax.

Signo de Cowen: Constricción de la pupila contralateral a sacudidas con la estimulación lumínica pupilar.

Signo de Dalmadez: Rigidez de los músculos faciales.

Signo de Dunphy: Inyección conjuntival por delante de la inserción del músculo recto externo.

Signo de Dalrymple: Ensanchamiento anormal de las fisuras palpebrales con retracción del párpado superior.

Signo de Enroth: Edema de los párpados, especialmente el superior cerca del margen supraorbitario.

Signo de Gifford: Dificultad para evertir el parpado superior.

Signo de Graefe: El párpado superior no sigue uniformemente el movimiento del globo ocular hacia abajo y queda la esclerótica blanca.

Signo de Grocco: Dilatación aguda del corazón luego de un esfuerzo importante en esta enfermedad.

Signo de Guttman: Retumbo que se escucha sobre la glándula tiroides.

Signo de Jellinek-Rasin: Pigmentación del párpado especialmente el superior.

Signo de Jendrassk-Brain: Parálisis de los músculos extraoculares.

Signo de Joffroy: Ausencia de arrugas en la frente en la mirada forzada hacia arriba.

Signo de Knies: Desigualdad en la dilatación pupilar.

Signo de Kocher: Mirada fija y atemorizadora.

Signo de Lian: Signo de hiperestesia cutánea. Para investigarlo se toma un alfiler, y en los casos de EG se limita una zona cutánea de hiperestesia que corresponde a la proyección de la glándula.

Signo de Locatello: La temperatura axilar es superior que la bucal en 0,3 a $0,3^{\circ}$ en estos pacientes.

Signo de Loewi: Dilatación fácil de la pupila con adrenalina. Signo de Mann: No parecen estar en el mismo nivel ambos ojos por disminución de la resistencia del cuero cabelludo.

Signo de Marañon: Retracción vasomotora tras la estimulación de la piel que recubre el cuello. 
Signo de Marañon: Frémito que experimenta la mano del observador al apoyarse ligeramente sobre el área tiroidea.

Signo de Marie: Temblor del cuerpo o de las extremidades.

Signo de Means-Griffith-Von Koller: Demora del párpado inferior al mirar hacia arriba.

Signo de Moebius: Falta de convergencia de los ejes oculares al mirar al observador acercándose.

Signo de Plummer: Debilidad de las piernas, que se evidencian al sentarse, o pasar de la posición de acostado a sentado.

Signo de Rosembach: Temblor fino de los parpados superiores con los ojos suavemente cerrados.

Signo de Rusell-Fraser: Disminución del surco entre el párpado superior y el globo ocular al cerrar los ojos.

Signo de Sainton: Contracción del músculo frontal, cuando el paciente mira hacia arriba.

Signo de Snellen-Riesman: Soplo que se escucha con el estetoscopio sobre el ojo cerrado.

Signo de Stellwag: Disminución en la frecuencia del parpadeo.
Signo de Suker: Fijación complementaria deficiente en la rotación hacia afuera.

Signo de Tellais: Pigmentación del párpado especialmente el inferior.

Signo de Topolanski: Congestión pericorneal del ojo.

Signo de Vigouroux: Disminución de la resistencia eléctrica de la piel.

Signo de Wilder: Leve contracción del globo ocular cuando se cambia su movimiento de abducción o aducción o viceversa.

A través de la historia, los médicos se han dedicado a encontrar pistas que conduzcan a un diagnóstico correcto en el caso de una patología tan común como la tiroidea $(1,4)$.

En el mundo de la medicina de hoy, en donde la tecnología juega un rol importante, como dice Alberto Agrest (9) "hemos llegado a escuchar poco, explicar menos, pedir muchos exámenes auxiliares y decidir sobre la base de lo que los exámenes nos informan", perdiendo así el valor relevante de la anamnesis y el examen físico.

\section{Bibliografía}

1. McKenna TJ. Graves' disease. Eponym. Lancet 2001; 357: 1793-6.

2. Coakley D. Robert Graves, evangelist of clinical medicine. Dublin: the Irish Endocrine Society 1996: 9-102.

3. Weetman AP. Graves' Disease. N Engl J Med 2000; 343: 1236-47.

4. Goring HD. Carl Adolph Von Basedow on the on the occasion of the $150^{\text {th }}$ anniversary of the day he died. J Dtsch Dermatol Ges 2004; 2 : 963-7.

5. Abalovich M, Alcaraz G, Gutierrez S, et al. Temas de Medicina Crítica y Terapia Intensiva. In: Solari LA. Corrales (eds). Emergencias Tiroideas. Buenos Aires: 1998, p 153-77.

6. Marañon G. Manual de Diagnóstico Etiológico. In: Espasa-Calpe editors. Bocio hipertiroideo. $5^{\text {th }}$ ed. Madrid: 1950, p 497-8.

7. Gaspary F. Semiotecnia y Fisiopatología. In: Mazzei ES, Rozman C. El Ateneo editors. Semiología de la Cabeza. Buenos Aires: 1980, p 10125 .

8. Lahey F. Apathetic thyroidism. Ann Surg 1931; 93: 1026-31.

9. Agrest A. Pasado, presente y futuro en la enseñanza de la medicina clínica. In: Cámera MI, Romani A, Madoery C, Farías J, editors. Avances en medicina '94. Buenos Aires: Sociedad Argentina de Medicina; 1994, p 295-306. 